\title{
Low bone mineral density in adults with cystic fibrosis
}

C S Haworth, P L Selby, A K Webb, M E Dodd, H Musson, R McL Niven, G Economou, A W Horrocks, A J Freemont, E B Mawer, J E Adams

Adult Cystic Fibrosis Unit, Wythenshawe Hospital, Manchester M23 9LT, UK

C S Haworth

A K Webb

M E Dodd

H Musson

R McL Niven

G Economou

A W Horrocks

\section{Department of}

Medicine, University

of Manchester,

Manchester Royal

Infirmary, Manchester

M13 9WL, UK

P L Selby

E B Mawer

Osteoarticular

Pathology,

Department of

Rheumatology

A J Freemont

Department of Diagnostic Radiology J E Adams

University of

Manchester,

Manchester M13 9PT, UK

Correspondence to:

Dr A K Webb.

Received 4 January 1999 Returned to authors 10 March 1999

Revised manuscript received

21 June 1999

Accepted for publication

24 June 1999

\section{Abstract}

Background-Patients with cystic fibrosis have several risk factors for the development of low bone mineral density (BMD). To identify the prevalence and clinical correlates of low BMD in adult patients with cystic fibrosis, densitometry was performed in 151 patients ( 83 men) aged $15-52$ years.

Methods-BMD was measured in the lumbar spine (L1-4) using dual energy $x$ ray absorptiometry (DXA) and quantitative computed tomography (QCT). It was also measured in the proximal femur (total hip and femoral neck) using DXA, and in the distal and ultra distal forearm using single energy $\boldsymbol{x}$ ray absorptiometry (SXA). Biochemical markers of bone turnover, vitamin D levels, parathyroid hormone levels, and a variety of anthropometric variables were also assessed.

Results-The mean (SD) BMD Z score was $-0.73(0.85)$ in the distal forearm, -0.31 (0.92) in the ultra distal forearm, -1.21 (1.18) in the lumbar spine using DXA, -0.56 (1.36) in the lumbar spine using QCT, -1.25 (1.30) in the femoral neck, and -1.01 (1.14) in the total hip. $34 \%$ of patients had a BMD $\mathrm{Z}$ score of $\mathbf{- 2}$ or less at one or more skeletal sites. Body mass index $(0.527, p=0.01)$, percentage predicted forced expiratory volume in one second $(0.388, p=0.01)$, and physical activity $(0.249, p=0.05)$ were positively related to the mean $\mathrm{BMD} Z$ score. Levels of $C$ reactive protein $(-0.328, p=$ $0.01)$, parathyroid hormone $(-0.311, \mathrm{p}=$ 0.01 ) and biochemical markers of bone turnover (osteocalcin -0.261 and bone specific alkaline phosphatase $-0.249, p=0.05$ ) were negatively related to the mean BMD $Z$ score. Vitamin $D$ insufficiency (25hydroxyvitamin $D<15 \mathrm{ng} / \mathrm{ml}$ ) was prevalent (53/139 patients, $38 \%)$ despite supplementation with 900 IU vitamin D per day.

Conclusions-Low bone density is prevalent in adult patients with cystic fibrosis. Current levels of vitamin D supplementation appear to be inadequate.

(Thorax 1999;54:961-967)

Keywords: cystic fibrosis; bone mineral density; osteoporosis; vitamin D insufficiency
Cystic fibrosis is the most common lethal autosomal recessive condition affecting the Caucasian population. The defective cystic fibrosis transmembrane regulator gene results in the clinical triad of chronic pulmonary sepsis, pancreatic insufficiency, and biliary cirrhosis. With improved treatment the median age of survival in the UK has risen to approximately 31 years. ${ }^{1}$ As a consequence of this increased survival, new and unusual complications of the disease are emerging, including low bone mineral density (BMD).

Patients with cystic fibrosis have several potential risk factors for the development of low BMD including calcium and vitamin $\mathrm{D}$ malabsorption, malnutrition, delayed puberty, hypogonadism, diabetes, reduced levels of physical activity, glucocorticoid therapy, cyclosporin therapy, and chronic pulmonary infection. ${ }^{2}$ The major clinical consequence of low BMD is fracture and recent evidence suggests that patients with cystic fibrosis have an increased fracture rate compared with the general population. ${ }^{34}$ Thoracic fractures are clinically significant because they hinder sputum clearance and reduce quality of life. In addition, some centres now consider symptomatic osteopenia to be a contraindication to transplantation.

The aim of this study was to identify the prevalence and clinical correlates of low BMD in a large group of adult patients with cystic fibrosis to facilitate the development of new treatment strategies.

\section{Methods}

SUBJECTS

All patients attending the Manchester Adult Cystic Fibrosis Unit were invited to participate in the study. One hundred and fifty one (83 men) of the 205 cystic fibrosis patients attending the unit were enrolled and the study was conducted between April and December 1997. The diagnosis of cystic fibrosis was confirmed by an elevated sweat chloride test, gene analysis, or an appropriate cystic fibrosis phenotype. The study was approved by the South Manchester ethics committee and each patient provided informed written consent. 
BONE DENSITY MEASUREMENTS

BMD was measured in the lumbar spine (L1-4) and proximal femur (femoral neck and total hip) by dual energy $x$ ray absorptiometry (DXA), (QDR 4500 Acclaim densitometer, Hologic, Waltham, Massachusetts, USA) and are measurements of integral (both cortical and trabecular) BMD. BMD was also measured in the distal and ultra distal forearm sites by single energy $x$ ray absorptiometry (SXA) (Osteometer DTX-100 densitometer, Osteometer Meditech, Roedovre, Denmark). The non-dominant forearm was used except when a history of fracture was obtained. The distal forearm measurement is predominantly of cortical $(87 \%)$ bone and the ultra distal forearm measurement is predominantly of trabecular $(65 \%)$ bone. BMD was also measured in the lumbar spine (L1-4) by quantitative computed tomography (QCT) (Picker PQ CT, Picker International, Stevenage, UK) using a CIRS (model IV) lumbar reference simulator (CIRS Inc, Norfolk, Virginia, USA). QCT is a measure of trabecular bone density.

DXA and SXA measure BMD in $\mathrm{g} / \mathrm{cm}^{2}$ while QCT measures true volumetric bone density in $\mathrm{g} / \mathrm{l}$. The departmental precision for each bone densitometry technique was derived from repeated measurements in 10 subjects. Expressed as a coefficient of variation, the departmental precisions are: DXA lumbar spine $1.1 \%$; femoral neck $3.3 \%$; total hip $1.3 \%$; SXA distal forearm $1.0 \%$; ultra distal forearm $2.5 \%$; and QCT $1.6 \%$.

The BMD results were expressed as $\mathrm{Z}$ scores (the number of SDs that a BMD measurement is from the mean of an age and sex matched control population) to minimise the effects of age and sex. The reference data used were those provided by the DXA and SXA densitometer manufacturers and by the manufacturers of the QCT lumbar reference simulator. Appropriate reference data were not available for patients under the age of 20 years, except for DXA measurements in the lumbar spine. $\mathrm{BMD}$ was judged to be reduced if the $\mathrm{Z}$ score was equal to or below -2 . $T$ scores (SDs in relation to sex matched peak BMD) were not

Table 1 Mean (SD) characteristics of patients

\begin{tabular}{llll}
\hline & Men $(n=83)$ & Women $(n=68)$ & Total group $(n=151)$ \\
\hline Age (years) & $25.4(6.4)$ & $25.1(8.0)$ & $25.3(7.1)$ \\
$\begin{array}{l}\text { Body mass index }\left(\mathrm{kg} / \mathrm{m}^{2}\right) \\
\begin{array}{l}\text { Forced expiratory volume in one } \\
\text { second (\% predicted) }\end{array}\end{array}$ & $51.1(2.5)$ & $21.3(2.4)$ & $21.2(2.5)$ \\
\hline
\end{tabular}

Table 2 Mean (SD) levels of bone biochemical markers

\begin{tabular}{lcl}
\hline & Mean $(S D)$ & Normal range \\
\hline Corrected calcium (mmol/l) & $2.4(0.09)$ & $2.10-2.65$ \\
Phosphate (mmol/l) & $1.1(0.2)$ & $0.7-1.4$ \\
Total alkaline phosphatase (IU/l) & $121.1(83.6)$ & $25-110$ \\
Osteocalcin (ng/ml) & $10.4(4.1)$ & $3.4-10.0$ \\
Bone specific alkaline phosphatase (U/l) & $20.9(9.8)$ & $10.0-23.0$ \\
Deoxypyridinoline crosslinks (nM/mM) & $7.4(5.1)$ & $2.3-7.4$ \\
25-hydroxyvitamin D (ng/ml) & $19.0(11.1)$ & $5-30$ \\
25-hydroxyvitamin D (nmol/l) & $48(28)$ & $13-75$ \\
1,25-dihydroxyvitamin D (pg/ml) & $31.6(12.8)$ & $20-50$ \\
1,25-dihydroxyvitamin D (pmol/l) & $76(31)$ & $48-120$ \\
Parathyroid hormone (pg/ml) & $26.1(14.3)$ & $10-60$ \\
\hline
\end{tabular}

used because the younger patients may not have reached their peak bone mass.

LABORATORY MEASUREMENTS

Morning non-fasting blood and urine samples were taken from patients on recruitment to the study at a time when their lung disease was stable. Serum samples were separated within 30 minutes of sampling and frozen at $-20^{\circ} \mathrm{C}$. Serum calcium, phosphate, total alkaline phosphatase, albumin, and $\mathrm{C}$ reactive protein (CRP) concentrations were measured with an AU600 automatic analyser (Olympus Diagnostic Systems, Eastleigh, Hampshire, UK). HbA1C was measured using a Mono-S Column (Pharmacia, Uppsala, Sweden). Serum vitamin D metabolites 25-hydroxyvitamin D (25-OHD) and 1,25-dihydroxyvitamin D $\left(1,25(\mathrm{OH})_{2} \mathrm{D}\right)$ were measured using in house assay systems as described previously, ${ }^{5}$ and the serum parathyroid hormone (PTH) concentration was measured using an Intact parathyroid hormone Allegro 100T kit (Nichols Institute Diagnostics, Saffron Walden, UK). Serum concentrations of osteocalcin, bone specific alkaline phosphatase (BSAP), urinary
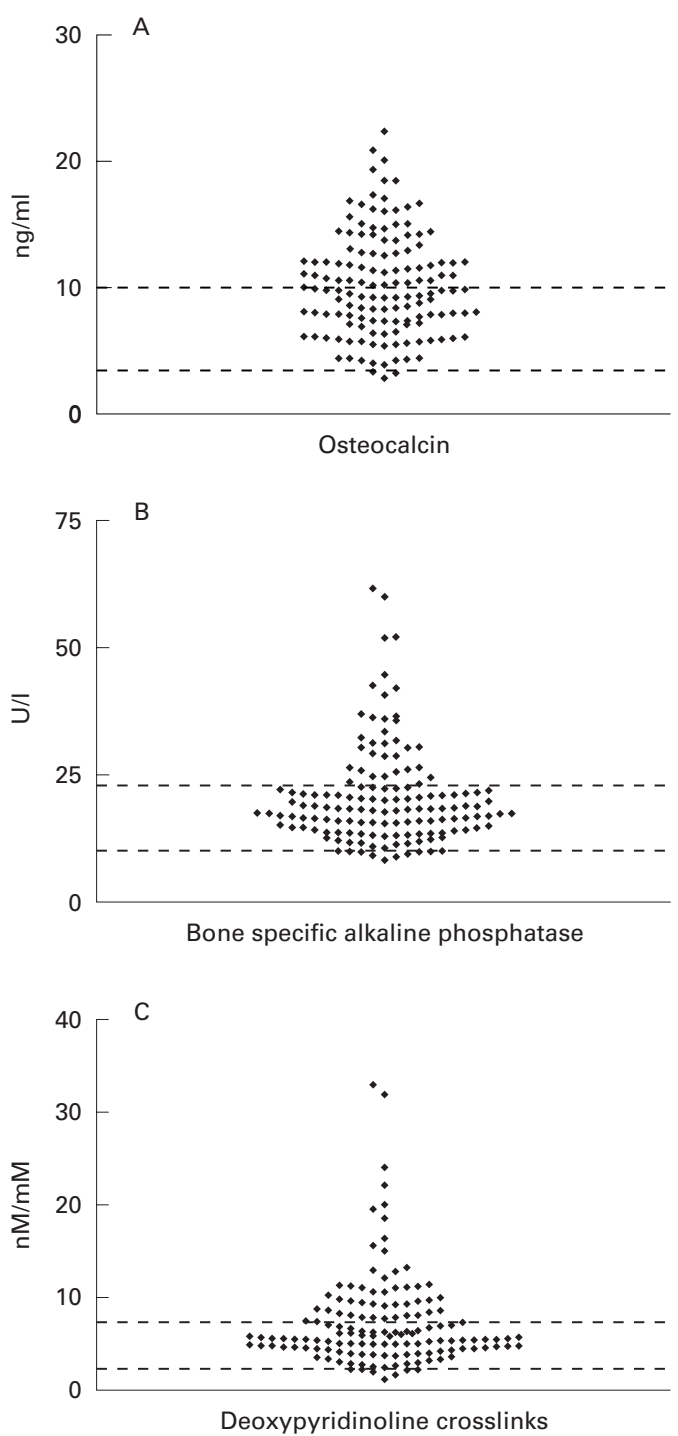

Figure 1 Serum levels of $(A)$ osteocalcin and $(B)$ bone specific alkaline phosphatase and $(C)$ urinary deoxypyridinoline crosslinks in adult patients with cystic fibrosis. Reference ranges indicated by horizontal lines. 

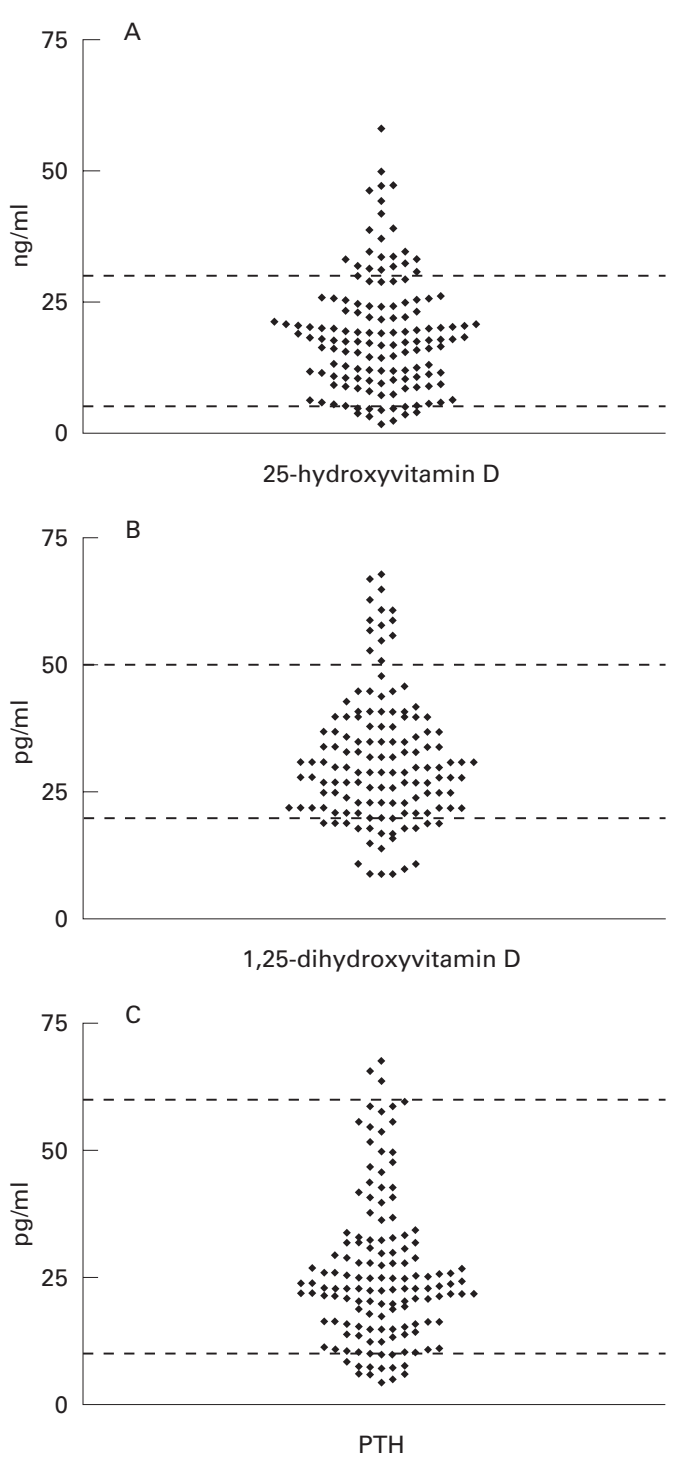

Figure 2 Serum levels of (A) 25-hydroxyvitamin D, (B) 1,25-dihydroxyvitamin $D$, and $(C)$ parathyroid hormone in adult patients with cystic fibrosis. Reference ranges indicated by horizontal lines.

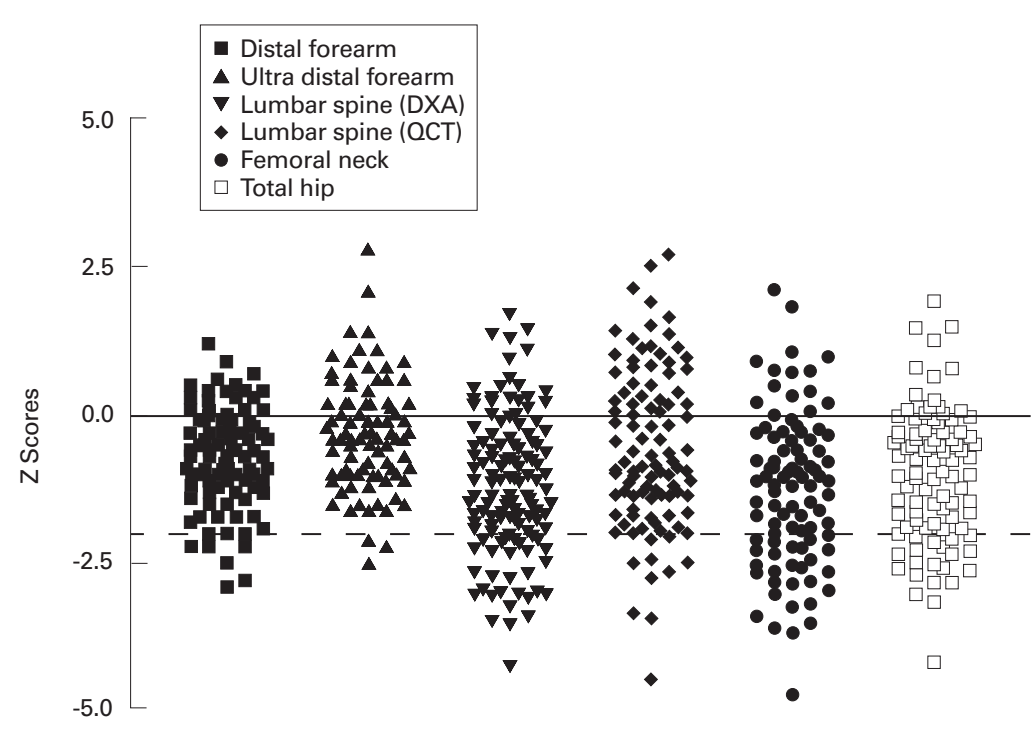

Figure 3 Bone mineral density $Z$ scores in adult patients with cystic fibrosis. The solid horizontal line indicates the mean bone mineral density $Z$ score of an age and sex matched control population and the broken horizontal line indicates a bone mineral density $Z$ score of -2 . deoxypyridinoline crosslinks and, in men, serum concentrations of testosterone, oestrogen, luteinising hormone (LH), and follicular stimulating hormone $(\mathrm{FSH})$ were measured using kits supplied by Metra Biosystems (UK) Ltd (Oxford, UK).

\section{CLINICAL MEASUREMENTS}

The best recorded forced expiratory volume in one second $\left(\mathrm{FEV}_{1}\right)$, weight, and height in the four months prior to recruitment were used to calculate the percentage predicted $\mathrm{FEV}_{1}{ }^{6}$ and body mass index (BMI; weight ( $\mathrm{kg}$ )/height $\left.(\mathrm{m})^{2}\right)$. Nutritional status was further assessed by calculating daily calorie intake from a three day food diary and daily calcium intake from a validated questionnaire. ${ }^{78}$ In female patients the age at menarche, menstrual history, and use of hormonal contraception were recorded. Patients were asked about previous fractures which had to have been confirmed radiographically. Levels of physical activity were assessed using the Baecke physical activity questionnaire which has been validated in young adults. ${ }^{9}$ The questionnaire documents the amount of physical activity performed during work, sport and leisure times, and each section is scored between 1 and 5, the total score ranging from a minimum of 3 to a maximum of 15 .

\section{STATISTICAL ANALYSIS}

Data analysis was undertaken using SPSS version 7.0 (SPSS Inc, Chicago, Illinois, USA). Since the BMD measurements at the different skeletal sites were significantly intercorrelated, principal components analysis (without rotation) was used to obtain summary measurements of BMD across skeletal sites. Principal components analysis is a technique that allows a large number of interrelated variables to be described by a smaller group of composite scores. ${ }^{10}$ The composite scores obtained from this procedure were then entered into further analyses to assess the relationships between disease severity, metabolic factors, and bone density.

Many of the markers of bone turnover and hormones involved in calcium homeostasis were not normally distributed, but for clarity all variables have been summarised using mean (SD) values. For all subsequent analysis non-parametric tests have been used.

\section{Results}

PATIENT CHARACTERISTICS

The characteristics of the patients are shown in table 1. None of the patients had had a transplant at the time of the study. Ninety one patients were homozygous and 47 were heterozygous for the delta F508 cystic fibrosis mutation. Nine patients had other mutations and the complete genotype of four patients has not been identified.

Thirteen patients required long term oral glucocorticoid treatment for either allergic bronchopulmonary aspergillosis or unstable lung disease. Ninety patients were prescribed inhaled corticosteroid therapy (budesonide 200-400 $\mu \mathrm{g}$ twice daily or fluticasone propionate $250-500 \mu \mathrm{g}$ twice daily). One patient was 
Table 3 Mean (SD) bone mineral density $Z$ scores

\begin{tabular}{llll}
\hline & $\begin{array}{l}\text { Men } \\
(n=79)\end{array}$ & $\begin{array}{l}\text { Women } \\
(n=64)\end{array}$ & $\begin{array}{l}\text { Total group } \\
(n=143)\end{array}$ \\
\hline Distal forearm $^{\star}$ & $-0.97(0.89)$ & $-0.39(0.66)$ & $-0.73(0.85)$ \\
Ultra distal forearm & $\mathrm{n}=52$ & $\mathrm{n}=38$ & $\mathrm{n}=90$ \\
& $-0.50(0.80)$ & $-0.04(1.01)$ & $-0.31(0.92)$ \\
Lumbar spine & $\mathrm{n}=52$ & $\mathrm{n}=38$ & $\mathrm{n}=90$ \\
Lumbar spine & $-0.85(1.18)$ & $-0.18(1.49)$ & $-0.56(1.36)$ \\
& $\mathrm{n}=56$ & $\mathrm{n}=43$ & $\mathrm{n}=99$ \\
Femoral neck $\ddagger$ & $-1.47(1.14)$ & $-0.90(1.16)$ & $-1.21(1.18)$ \\
Total hip $\ddagger$ & $\mathrm{n}=68$ & $\mathrm{n}=57$ & $\mathrm{n}=125$ \\
& $-1.40(1.34)$ & $-1.06(1.20)$ & $-1.25(1.30)$ \\
& $\mathrm{n}=55$ & $\mathrm{n}=39$ & $\mathrm{n}=94$ \\
& $-1.18(1.18)$ & $-0.77(1.03)$ & $-1.01(1.14)$ \\
& $\mathrm{n}=55$ & $\mathrm{n}=39$ & $\mathrm{n}=94$ \\
\hline
\end{tabular}

${ }^{\star}$ Measured by single energy $x$ ray absorptiometry.

†Measured by quantitative computed tomography.

$\ddagger$ Measured by dual energy $x$ ray absorptiometry.

on azathioprine for co-existing inflammatory bowel disease and one was on cyclosporin for the treatment of recurrent cystic fibrosis related vasculitis.

Nine patients were pancreatic sufficient. All pancreatic insufficient patients were prescribed $22.5 \mu \mathrm{g}$ (900 IU) vitamin D (calciferol) daily, but 20 patients admitted to not taking it on a regular basis. One patient had been taking calcium supplements and alendronate for approximately 12 months.

The mean (SD) age at menarche was 13.9 (1.6) years. Two female patients aged 16 and 18 years had primary amenorrhoea. One patient had had secondary amenorrhoea for 15 months and had a BMI of 16.6. Two patients reported menstrual irregularity (having fewer than nine periods per year), one of whom was perimenopausal. One patient was postmenopausal and on hormone replacement therapy. Twenty three patients reported taking the combined oral contraceptive pill or depot medroxyprogesterone acetate.

Twenty six patients had cystic fibrosis related diabetes, 21 of whom required insulin therapy and five were taking oral hypoglycaemic agents. Nine patients admitted to smoking regularly and 26 patients admitted to consuming 20 or more units of alcohol per week. The mean (SD) physical activity score was 7.6 (1.4). Sixteen patients reported a family history of osteoporosis.

Fifty one of the 151 patients (34\%) reported a history of fracture. The forearm was the most common site.

Analysis of the three day food diaries ( $\mathrm{n}=$ 63) revealed that the mean (SD) daily energy intake as a percentage of the reference nutrient intake (RNI) was 138 (35)\%. The mean daily calcium intake $(\mathrm{n}=134)$ was $1450(839) \mathrm{g}$.

Table 4 Mean (SD) bone turnover marker levels, vitamin D levels, and parathyroid hormone levels in delta F508 homozygote and non-delta F508 homozygote cystic fibrosis adult patients

\begin{tabular}{lccl}
\hline & $\begin{array}{l}\text { Delta F508 } \\
\text { homozygotes } \\
(n=90)\end{array}$ & $\begin{array}{l}\text { Non-delta F508 } \\
\text { homozygotes } \\
(n=57)\end{array}$ & p value \\
\hline Osteocalcin (ng/ml) & $10.6(4.2)$ & $10.0(3.9)$ & NS \\
Bone specific alkaline phosphatase $(\mathrm{U} / \mathrm{l})$ & $22.5(11.1)$ & $18.1(6.9)$ & 0.01 \\
Deoxypyridinoline crosslinks $(\mathrm{nM} / \mathrm{mM}$ creatinine) & $7.9(5.3)$ & $6.2(4.6)$ & 0.005 \\
25-hydroxyvitamin D (ng/ml) & $17.2(9.0)$ & $22.0(13.5)$ & $\mathrm{NS}$ \\
1,25-dihydroxyvitamin D (pg/ml) & $31.5(11.7)$ & $32.7(14.4)$ & $\mathrm{NS}$ \\
Parathyroid hormone $(\mathrm{pg} / \mathrm{ml})$ & $27.0(14.2)$ & $24.3(13.6)$ & $\mathrm{NS}$ \\
\hline
\end{tabular}

LABORATORY MEASUREMENTS

Serum and urine samples were collected from 139 of the 151 patients. The mean (SD) levels of bone biochemical markers are summarised in table 2. In addition, the bone turnover marker levels are shown graphically in fig $1 \mathrm{~A}-\mathrm{C}$ and the vitamin D levels and PTH levels are presented in fig $2 \mathrm{~A}-\mathrm{C}$. Ten patients were vitamin $\mathrm{D}$ deficient with 25-OHD levels below the reference range, while 20 patients had subnormal levels of $1,25(\mathrm{OH})_{2} \mathrm{D}$ and 14 patients had increased levels of $1,25(\mathrm{OH})_{2} \mathrm{D}$. Eleven patients had subnormal PTH concentrations and in three the concentrations were above normal.

The mean (SD) levels of testosterone, oestrogen, $\mathrm{LH}$, and FSH in the male patients were $5.1(2.0) \mathrm{ng} / \mathrm{ml}$ (reference range 2.8-8.2), $14.6(4.9) \mathrm{pg} / \mathrm{ml}$ (reference range 10-40), 3.5 (1.7) $\mathrm{mU} / \mathrm{ml}$ (reference range 1.3-6.1), and $1.6(1.1) \mathrm{mU} / \mathrm{ml}$ (reference range $1.2-11.0$ ), respectively.

The mean (SD) levels of CRP, serum albumin, and HbA1C were 24.2 (36.5) $\mathrm{mg} / \mathrm{l}$, 41.1 (3.8) g/l, and $5.2(1.5) \%$, respectively.

BONE MINERAL DENSITY MEASUREMENTS

BMD data are presented for 143 of the 151 patients enrolled in the study. Appropriate reference data were unavailable for eight patients who were under 20 years of age and consented to QCT bone densitometry only. A BMD Z score of -2 or less at one or more skeletal sites was shown by 48 of the 143 patients (34\%; fig $3)$. The mean (SD) BMD Z score at each skeletal site is shown in table 3 .

The first principal component derived from the BMD measurements was a linear combination of the BMD $\mathrm{Z}$ scores at all skeletal sites. These were weighted by the principal components analysis, although the weighting coefficients were similar (ranging from 0.69 for the distal forearm to 0.89 for the total hip). In effect, therefore, this component is best thought of as a mean BMD Z score and it accounted for $68.2 \%$ of the variance of BMD.

The second principal component gave information about the difference in BMD between the forearm sites (which had positive coefficients) and axial sites (with negative coefficients). This accounted for a further $14.8 \%$ of the variance.

The third principal component reflected the difference between BMD in the spine (by both DXA and QCT with coefficients of 0.29 and 0.49 , respectively) and the two hip sites (femoral neck coefficient -0.40 and total hip -0.31 ). Neither forearm measurements made any significant contribution to this component, which accounted for $9.7 \%$ of the variance. None of the other principal components accounted for more than $5 \%$ of the variance in BMD.

The mean BMD $\mathrm{Z}$ score was significantly lower in men than in women $(p=0.02)$. There was no significant difference in the mean BMD $\mathrm{Z}$ score between women receiving hormonal contraception and those who were not $(\mathrm{p}=$ $0.91)$.

There was no significant difference in the mean BMD Z score between patients who were 
Table 5 Correlates of bone mineral density (BMD)

\begin{tabular}{|c|c|c|c|}
\hline & $\begin{array}{l}\text { Factor } 1 \\
\text { (mean BMD } \\
Z \text { score) }\end{array}$ & $\begin{array}{l}\text { Factor } 2 \\
\text { (forearm vs } \\
\text { axial BMD) }\end{array}$ & $\begin{array}{l}\text { Factor } 3 \\
\text { (lumbar spine } \\
\text { vs hip BMD) }\end{array}$ \\
\hline Age & -0.026 & $0.296^{\star \star}$ & 0.090 \\
\hline Body mass index & $0.527^{\star \star}$ & -0.060 & -0.131 \\
\hline $\begin{array}{l}\text { Forced expiratory volume in one second } \\
(\% \text { predicted })\end{array}$ & $0.388^{\star \star}$ & $-0.284^{\star}$ & -0.161 \\
\hline Physical activity & $0.249^{\star}$ & $-0.278^{\star}$ & -0.092 \\
\hline Calcium intake & -0.065 & -0.084 & $-0.321^{\star \star}$ \\
\hline$\%$ reference nutrient intake & -0.093 & 0.027 & $-0.399^{\star \star}$ \\
\hline Albumin & 0.067 & -0.153 & -0.107 \\
\hline $\mathrm{HbA} 1 \mathrm{C}$ & -0.034 & -0.005 & 0.062 \\
\hline $\mathrm{C}$ reactive protein & $-0.328^{\star \star}$ & 0.187 & 0.018 \\
\hline Osteocalcin & $-0.261^{\star}$ & -0.160 & 0.011 \\
\hline Bone specific alkaline phosphatase & $-0.249^{\star}$ & -0.011 & -0.065 \\
\hline Deoxypyridinoline crosslinks & -0.135 & 0.009 & 0.184 \\
\hline 25-hydroxyvitamin D & 0.085 & -0.047 & 0.106 \\
\hline 1,25-dihydroxyvitamin D & -0.149 & -0.008 & $0.358^{\star \star}$ \\
\hline Parathyroid hormone & $-0.311^{\star \star}$ & 0.128 & 0.179 \\
\hline Corrected calcium & 0.021 & 0.126 & $-0.228^{\star}$ \\
\hline Phosphate & 0.066 & 0.029 & -0.146 \\
\hline Total alkaline phosphatase & $-0.255^{\star}$ & 0.138 & -0.131 \\
\hline
\end{tabular}

homozygous for the delta F508 mutation and those who were not $(p=0.75)$. However, the mean BSAP levels and urinary deoxypyridinoline crosslinks were significantly different between the two groups, with the homozygotes having higher levels of bone turnover than the non-homozygotes (table 4).

There was no significant difference in the mean BMD Z score between those patients with and without a history of fracture $(\mathrm{p}=$ $0.63)$.

The clinical and biochemical correlates of the BMD measurements are recorded in table 5. There was a significant positive relationship between the mean BMD $\mathrm{Z}$ score (the first principal component) and $\mathrm{BMI}, \mathrm{FEV}_{1}$ percentage predicted, and physical activity. There was a significant negative relationship between the mean BMD $\mathrm{Z}$ score and serum levels of CRP, osteocalcin, BSAP, PTH, and total alkaline phosphatase.

The difference between the forearm and axial BMD (the second principal component) increased with age and was negatively related to both respiratory function, as indicated by the $\mathrm{FEV}_{1}$ percentage predicted, and the level of physical activity.

The difference between the lumbar spine and hip BMD (the third principal component) appeared predominantly to reflect nutritional status, being positively related to $1,25(\mathrm{OH})_{2} \mathrm{D}$ levels and being negatively related to calcium intake, percentage RNI, and serum corrected calcium.

\section{Discussion}

This is the largest and most comprehensive study to date investigating the prevalence and causes of low BMD in adults with cystic fibrosis. Thirty four percent of patients had a BMD $\mathrm{Z}$ score of -2 or less, which suggests that these individuals have at least a threefold increased risk of fracture compared with individuals of the same age and sex. ${ }^{11}$ The altered nutrition and small body size of patients with cystic fibrosis might have influenced BMD measurements using DXA, but the fact that similar changes were seen with QCT, which gives a volumetric measure of $\mathrm{BMD}$, indicates that this was not a significant effect.

The results of earlier studies documenting $\mathrm{BMD}$ in adults with cystic fibrosis involve relatively small numbers of patients and diverse study populations. Aris et al measured the BMD of 70 adults with cystic fibrosis referred for lung transplantation. ${ }^{3}$ The mean BMD results in the spine, proximal femur, and total body were approximately 2 SDs below those in age matched controls. Donovan et al studied 30 patients with cystic fibrosis awaiting lung transplantation. ${ }^{12}$ The mean BMD Z scores in women and men were between 1 and 2 SDs below normal at axial sites and somewhat less reduced in the radius. Both these studies involved subjects with more severe respiratory disease than reported here and this might explain the differences between these results and ours. Bhudhikanok et al studied 49 patients with cystic fibrosis aged between eight and 48 years, and the mean BMD $\mathrm{Z}$ score was -1.7 in the lumbar spine and -1.9 in the femoral neck. ${ }^{13}$ More than half of these patients required alternate day or daily glucocorticoid therapy and this might explain the lower BMD in this cohort than in ours. Grey et al studied 16 adults with cystic fibrosis of moderate severity and found that their BMD was reduced by $12.5 \%$ in the lumbar spine $(p<0.001)$ and by $11.1 \%$ in the femoral neck $(\mathrm{p}<0.001)$ compared with young normal controls. ${ }^{14}$ The current study involves a large and heterogeneous group of adults with cystic fibrosis and the $\mathrm{BMD}$ results may therefore be different from those derived from smaller, more specific study populations - for example, patients with cystic fibrosis awaiting lung transplantation. However, contrasting results could also arise from the fact that intercontinental differences in normal bone density may exist and some studies have employed locally derived reference data whereas others have used reference data provided by the densitometer manufacturers.

Although principal components analysis is not commonly used for the analysis of BMD values, they are ideally suited to this technique. ${ }^{10}$ The use of principal components analysis has clarified relationships that were less marked if the bone measurements had been considered independently at each skeletal site. Furthermore, the three significant principal components have meaningful biological correlates. The first component (mean BMD Z score) represents the effects on the skeleton irrespective of site, the second component demonstrates the contrasting behaviour of the forearm and axial skeleton, which is well recognised in other malabsorption syndromes, ${ }^{13}$ and the third principal component demonstrates the contrasting bony characteristics of the lumbar spine and proximal femur.

BMD was significantly lower in men than in women. They had comparable lung disease and nutritional indices and there was no evidence of male hypogonadism, so these differences are not easily explained. There was no relationship between the mean BMD $\mathrm{Z}$ score and age. Studies investigating BMD in children, two of 
which used QCT, have shown that BMD is already reduced in children with cystic fibrosis. ${ }^{16}{ }^{17}$ Poor bone accretion in childhood may therefore be partly responsible for the deficits in BMD affecting the adult population. However, the fundamental change in dietary advice in the late 1980s following the observations of Corey et al and the introduction of enteric coated pancreatic enzyme supplements may have resulted in improved bone accretion in paediatric patients over the last decade. ${ }^{18}$

Body mass index was positively related to $\mathrm{BMD}$, which is consistent with several other studies. ${ }^{32-14}$ Explanations for this include a direct effect of nutrition on the skeleton and an indirect effect via skeletal loading.

Physical activity correlated positively with BMD, which suggests that weight bearing activity may be an important determinant of BMD.

The severity of cystic fibrosis disease, indicated by the percentage predicted $\mathrm{FEV}_{1}$, was positively related to BMD and this is consistent with some, ${ }^{13}{ }^{14}$ but not all, ${ }^{312}$ studies in adult patients. The lack of correlation between $\mathrm{BMD}$ and $\mathrm{FEV}_{1}$ in these latter studies probably reflects the fact that all of the patients had severe lung disease and were awaiting lung transplantation. The exact mechanisms by which the severity of cystic fibrosis influences BMD have not been fully elucidated. Patients with more severe disease are often nutritionally depleted, less active, and may require more glucocorticoid therapy, all of which may affect BMD. An alternative theory suggests that inflammatory cytokines such as tumour necrosis factor $\alpha$, interleukin 1 , and interleukin 6 , which are increased in patients with cystic fibrosis due to chronic pulmonary infection, affect osteoblast and osteoclast activity and result in reduced BMD. ${ }^{314}$ This theory is supported by the significant negative relationship between CRP levels and BMD in the current study.

There was significant correlation between many of the different indicators of severity of cystic fibrosis such as percentage predicted $\mathrm{FEV}_{1}, \mathrm{BMI}$, and CRP (data not shown). It is therefore difficult to determine whether the observed relationships are indeed the result of changes in the variable in question or whether they reflect an overall change in disease severity. Since the distribution of the variables departed so far from normality, it was not possible to dissect out these relationships using multiple regression through transformation of the data. Since it is not possible to infer causation from correlation in any case, it is important that these relationships are tested in a longitudinal study.

Most of the vitamin D and PTH levels were within the normal ranges. However, the reference ranges for these variables are extremely wide as they apply to all age groups including the elderly. Considering the young age of the study population and the fact that they were prescribed 900 IU of vitamin D per day, these results show frank vitamin D deficiency in 10 of 139 patients (7\%) - that is, 25-OHD levels associated with osteomalacia.
Thirty patients (22\%) had levels below $10 \mathrm{ng} /$ $\mathrm{ml}$ and $53(38 \%)$ were below $15 \mathrm{ng} / \mathrm{ml}$, a value currently taken as indicating vitamin D insufficiency. ${ }^{19}$ This is consistent with the findings of Donovan and colleagues who reported a high proportion of patients with low 25-OHD levels and associated this with a significant reduction in $\mathrm{BMD}^{12}$ In common with others, ${ }^{313142021}$ we failed to show a direct association between vitamin $\mathrm{D}$ levels and BMD, but we did find a significant negative relationship between PTH levels and the mean BMD Z score. PTH may therefore be a more sensitive marker of vitamin $D$ insufficiency in this population. The suggestion that current vitamin $\mathrm{D}$ supplementation is inadequate is supported further by the findings of Hanly et al who reported that oral vitamin D supplementation ( $800 \mathrm{IU} /$ day for $4-10$ weeks) failed to achieve normal 25-OHD levels in $40 \%$ of patients with cystic fibrosis. ${ }^{22}$ The contrasting vitamin $\mathrm{D}$ results between studies may be explained by the different assay methods used, the size and disease severity of the study populations, adherence to vitamin D supplementation, dietary vitamin $\mathrm{D}$ intake, and patterns of solar exposure. It is striking that so many patients had suboptimal 25-OHD levels when it is well known that the majority of the vitamin $\mathrm{D}$ in the normal population arises from sunlight and not the diet, the British diet supplying only about $100 \mathrm{IU}$ per day. This suggests that the patients were not receiving normal sunlight exposure.

Bone turnover was increased in a large proportion of patients, which may partly reflect growth in the younger participants. However, there was a significant negative relationship between the markers of bone formation and the mean BMD $\mathrm{Z}$ score, which suggests that the patients with low bone density tended to have high bone turnover. Baroncelli et al studied bone turnover in young adults with cystic fibrosis and age matched controls. Analysis suggested an imbalance of bone resorption over bone formation in the cystic fibrosis group resulting in net bone loss. ${ }^{23}$ The significant differences in bone turnover between delta F508 homozygotes and non-homozygotes also suggests that there may be a genetic component influencing bone and calcium homeostasis in patients with cystic fibrosis.

A limitation of this study is that it does not address the influence of glucocorticoid therapy on BMD in adults with cystic fibrosis. The role of glucocorticoids in the development of low BMD is likely to be important, as in other chest diseases, but can only be properly addressed in a prospective study. It is proposed to use the current database as a baseline for such a prospective study. In addition, the precise histomorphometric features of cystic fibrosis bone are currently unknown. The observed reductions in BMD have been attributed to osteoporosis, but may represent a more complex metabolic bone disease, particularly as cystic fibrosis patients have pathology involving the gastrointestinal, pancreatic, and biliary systems. Labelled bone biopsies are required to 
further our understanding of cystic fibrosis related bone disease. ${ }^{24}$

In conclusion, BMD is significantly reduced in adult patients with cystic fibrosis and is related to markers of disease severity such as $\mathrm{BMI}, \mathrm{FEV}_{1}, \mathrm{CRP}$, and physical activity. Current levels of vitamin D supplementation appear to be inadequate and the differences in bone turnover between delta F508 homozygotes and non-homozygotes suggests that there may be a genetic component to the aetiology of low BMD in adults with cystic fibrosis.

This study was supported by a grant from the Cystic Fibrosis Middleton, M Hodgkinson, C Alsop, D Ledgerton, and R LarMiddleton, M Hodgkinson, C Alsop, D Ledgerton, and R Larder for performing the bone densitometry measurements. We would like to thank Professor Graham Dunn (Professor of
Biostatistics, Manchester University) for his invaluable statistical advice.

1 Shale DJ. Predicting survival in cystic fibrosis. Thorax 1997;52:309.

2 Haworth CS, Selby PL, Webb AK, et al. Osteoporosis in adults with cystic fibrosis. $\mathcal{F} R$ Soc Med 1998;91(Suppl 34): 14-8.

3 Aris RM, Renner JB, Winders $\mathrm{AD}$, et al. Increased rate of fractures and severe kyphosis: sequelae of living into adulthood with cystic fibrosis. Ann Intern Med 1988;128:186hood

4 Henderson RC, Specter BB. Kyphosis and fractures in children and young adults with cystic fibrosis. I Pediatr dren and young

5 Mawer EB, Walls J, Howell A, et al. Serum 1,25dihydroxyvitamin $\mathrm{D}$ may be related inversely to disease activity in breast cancer patients with
Clin Endicrinol Metab 1997;82:118-22.

6 Knudson RJ, Lebowitz MD, Hollberg CJ, et al. Changes in the normal maximum expiratory flow volume curve with growth and ageing. Am Rev Respir Dis 1983;127:725-34.

7 Basiotis P, Welsh SO, Cronin FJ, et al. Number of days of food intake records required to estimate individual and group nutrient intakes with defined confidence. $\mathcal{F}$ Nut 1987;117:1638-41.
8 Nelson M, Hague GF, Cooper C, et al. Calcium intake in the elderly: validation of a dietary questionnaire. $\mathcal{F}$ Hum Nutr Dietet 1988;1:115-27.

9 Baecke JAH, Burema J, Frijters JER. A short habitual questionnaire for the measurement of habitual physical activity in epidemiological studies. Am f Nutr 1982;36:936-42.

10 Everitt BS. Statistical methods for medical investigations. London: Edward Arnold, 1989: 100-10.

11 Cummings SR, Black DM, Nevitt MC, et al. Bone density at various sites for the prediction of hip fractures. Lancet 1993;341:72-5.

12 Donovan DS, Papadopoulos A, Staron RB, et al. Bone mass and vitamin $\mathrm{D}$ deficiency in adults with advanced cystic fibrosis lung disease. Am F Respir Crit Care Med 1998;158 1892-9.

13 Bhudhikanok GS, Lim J, Marcus R, et al. Correlates of osteopenia in patients with cystic fibrosis. Pediatrics 1996;97:103-11.

14 Grey AB, Ames RW, Matthews RB, et al. Bone mineral density and body composition in adult patients with cystic fibrosis. Thorax 1993;48:589-93.

15 Selby PL, Davies M, Adams JE, et al. Bone loss in celiac disease is related to secondary hyperparathyroidism. F Bone Min Res 1999;14:652-7.

16 Gibbens DT, Gilsanz V, Boechat MI, et al. Osteoporosis in cystic fibrosis. F Pediatr 1988;113:295-300.

17 Shaw N, Bedford C, Heaf D, et al. Osteopenia in adults with cystic fibrosis. Am f Med 1995;99:690-1.

18 Corey M, McLaughlin FJ, Williams M, et al. A comparison of survival, growth, and pulmonary function in patients with cystic fibrosis in Boston and Toronto. 7 Clin Epidemiol 1988;41:583-91.

19 Thomas MK, Lloyd-Jones DM, Thadhani RI, et al. Hypovitaminosis D in medical inpatients. N Engl F Med 1998;338 777-83.

20 Bachrach LK, Loutit CW, Moss RB, et al. Osteopenia in adults with cystic fibrosis. Am f Med 1994;96:27-34.

21 Stead RJ, Houlder S, Agnew J, et al. Vitamin D and parathyroid hormone and bone mineralisation in adults with cystic fibrosis. Thorax 1988;43:190-4.

22 Hanly JG, McKenna MJ, Quigley C, et al. Hypovitaminosis $\mathrm{D}$ and response to supplementation in older patients with cystic fibrosis. $O f$ Med 1985;219:377-85.

23 Baroncelli GI, De Luca F, Magazzu G, et al. Bone demineralization in cystic fibrosis: evidence of imbalance between bone formation and degradation. Pediatr Res 1997;41:397403.

24 Haworth CS, Selby PL, Webb AK, et al. Severe bone pain after intravenous pamidronate in adult patients with cystic fibrosis. Lancet 1998;352:1753-4. 